\title{
A bibliometric analysis of science and technology publication output of University of Electronic and Technology of China in Science Citation Index Expanded (SCI-E), 2003-2012
}

\author{
Yang WEN $^{1, a}$ \\ ${ }^{1}$ Library of University of Electronic Science and Technology of China, Chengdu 611731, China \\ aweny@uestc.edu.cn
}

\begin{abstract}
The main focal point of this paper lies on the appraisal of rising science and technology (S\&T) output in University of Electric science and Technology of China (UESTC) with the help of bibliometric indicators. The present study is a bibliometric assessment of S\&T output of UESTC, Which describes the growth, contribution and impact of research carried out by the faculty members, researchers or students of UESTC. It also attempts to analyze the growth and development of research activity of the university as reflected in publications output covered by Science Citation Index Expanded (SCI-E) during the period under study. This study presents the analysis of 8050 publications that were published in different SCI-E periodicals between 2003 and 2012, focusing on four aspects of the SCIE publication during 2003 to 2012, thus, the annual average growth rate percent, authorship pattern, collaborative country and scientific disciplines.
\end{abstract}

Keyword: Bibliometrics analysis; Science Citation Index Expanded (SCI-E); publication output; UESTC

\section{Introduction}

Bibliometric analysis is currently used for evaluating the qualitative and quantitative interest in a specific field through the analysis of publications. This method is currently used in the sciences of information to describe patterns of publications within a given field. Bibliometrics refers to research methodology employed in library and information sciences, which utilizes quantitative analysis and statistics methods to describe distribution patterns of articles with a given topic (Almind and Ingwersen, 1997), field (Campanario et. al.,
2006), institute (Moed et. al., 1985) or country (Schubert et al., 1989). These methods have been used to investigate research trends of specific fields recently (Vergidis et. al., 2005; Falagas et. al., 2006; Kumari, 2006).

Global landscape of Science, Technology and Innovation is changing. The aim of this paper is not only to provide the stand of UESTC, a university, in the world of S\&T in terms of scientific productivity and its impact in the S\&T society, but also to flash out possible fundamental ingredients for further research. Bibliometric analysis of S\&T output is a process, not just a result.

\section{Material and methods}

This paper is designed based on the bibliometric analysis. The online version of the Science Citation Index Expanded (SCI-E) of ISI Web of Science of UESTC was explored for the period 2003-2012. All publication types were included, through an advanced search in the platform web of knowledge, using the algorithm: $\mathrm{AD}=$ ((( univ* elec* sci* tech*) same (chengdu 610054 or $611731))$ or uestc or (uest same china) or ((610054 or 611731) same univ*)) or ((univ* elec* sci* tech*) not (xian or guilin or hangzhou) )) and Databases = SCI-E Timespan $=01 / 01 / 2003-12 / 31 / 2012$. A total of publication records were downloaded for this analysis in 1 November, 2014.

Further, the result was refined only to 8050 publication records. Finally 8050 research publication in different disciplines of science and technology retrieved matched with UESTC as author's affiliation/address. Aspects referring to type of document, different disciplines, collaborative country, citation and h-index were analyzed 
with MS-Excel.

\section{Results and Analysis}

\subsection{Details of publication}

A total of 8050 publications of UESTC are indexed in Science Citation Index Expanded (SCI-E) of ISI Web of
Science during 2003-2012. Table1 gives a detailed overview of publications with their citations details as 8050 papers are totally 54698 times cited, 47481 times cited without self-citations with an average of 6.79 citations per paper and h-index of 65 .

Table1 Detail of publication

\begin{tabular}{|l|l|}
\hline Total results & 8050 \\
\hline Sum of times cited & 54698 \\
\hline Sum of times cited without self-citations & 47481 \\
\hline Citing articles & 39455 \\
\hline Citing articles without self-citations & 36086 \\
\hline Average citations per item & 6.79 \\
\hline H-index & 65 \\
\hline
\end{tabular}

\subsection{Annual distribution of publication}

This section provides a thorough analysis of the annual distribution of publications of UESTC during 2003-2012. As depicted in Fig. 1, an increase in publication output over the given period can be seen. If we compare the output in the year 2003 with that in 20012, we can clearly see that, the number of publications has almost more ten times. Its annual growth rate is average $31.36 \%$.
Quite apart from the fact that there were some fluctuations in the number of publications every year, an increasing trend can be well observed. The publication productivity of UESTC grew quite significantly, showing the largest increases that UESTC were at $106.43 \%$ and $55.56 \%$ for 2004 and 2006 respectively. The largest number of publications was produced in the year 2012, with an increase of $24.6 \%$.

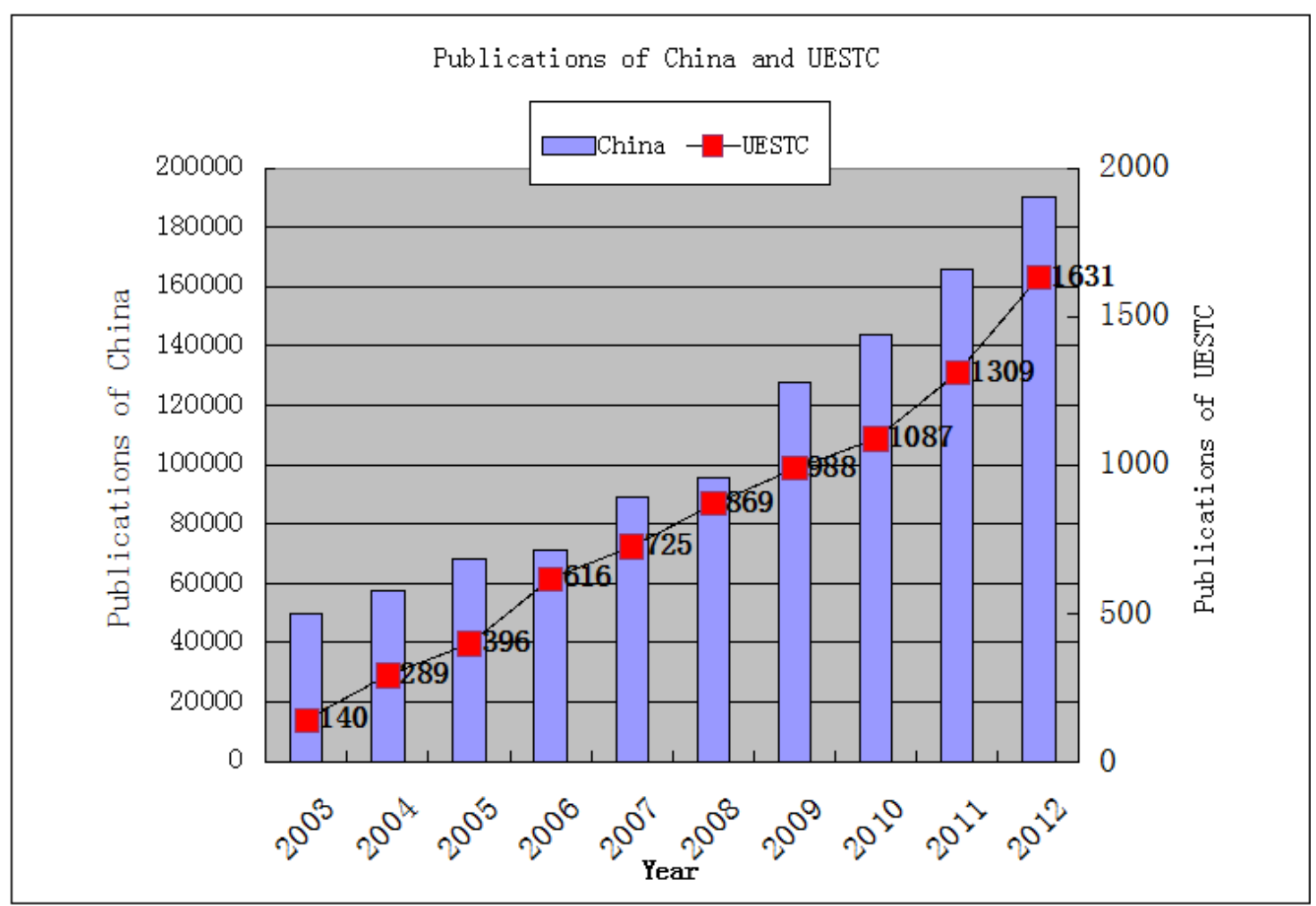

Fig.1 Trend of annual publications of UESTC 


\subsection{Authorship pattern of publications}

Derek John De Solla Price (1963) came up with a work called "Little Science, Big Science" where he explained the trend of and highly appreciated scientific research collaboration among authors. He also presaged that "by 1980 the single - author paper will be extinct." Such trends characterize both the social and the cognitive structure of scientific research realms (Glänzel, 2002). Collaborative research is common feature which is seen in science field especially during the 21 st century. It is a natural reflection of complexity, scale and costs of modern investigations in S\&T.

Table 2 provides the overview of authorship pattern of publications of UESTC during 2003-2012. $t$ is observed that just $2.31 \%$ of total papers involved single authors, two authors (13.18\%), three authors (24.62\%), and an astonishing $59.89 \%$ of papers were four and more than four authors. Multi authorship provides different measures of collaboration in science research and multi-authored papers were dominant over single-authored papers.

Table 2 Authorship pattern of publications of UESTC

\begin{tabular}{|c|c|c|c|c|c|}
\hline Year & One & Two & Three & $\geq$ Four & Total \\
\hline 2003 & 9 & 31 & 35 & 65 & 140 \\
\hline 2004 & 10 & 67 & 87 & 125 & 289 \\
\hline 2005 & 8 & 73 & 116 & 199 & 396 \\
\hline 2006 & 22 & 92 & 153 & 349 & 616 \\
\hline 2007 & 19 & 121 & 188 & 397 & 725 \\
\hline 2008 & 23 & 109 & 231 & 506 & 869 \\
\hline 2009 & 22 & 121 & 241 & 604 & 988 \\
\hline 2010 & 20 & 121 & 296 & 650 & 1087 \\
\hline 2011 & 22 & 150 & 281 & 856 & 1309 \\
\hline 2012 & 31 & 176 & 354 & 1070 & 1631 \\
\hline Grand Total & $186(2.31)$ & $1061(13.18)$ & $1982(24.62)$ & $4821(59.89)$ & 8050 \\
\hline
\end{tabular}

Note: figures in parentheses represented percentage.

\subsection{Collaborative Country}

In 2003-2012, UESTC carried out international co-publication with 46 countries and regions and collaborative country is mainly in developed country. Among them, the number of international co-publication with United States is the most and reaches 850 during ten years, accounting for $48.13 \%$ of total international co-publication of UESTC and is far higher than with Singapore in the second. The number of international co-publication with Singapore is 277.

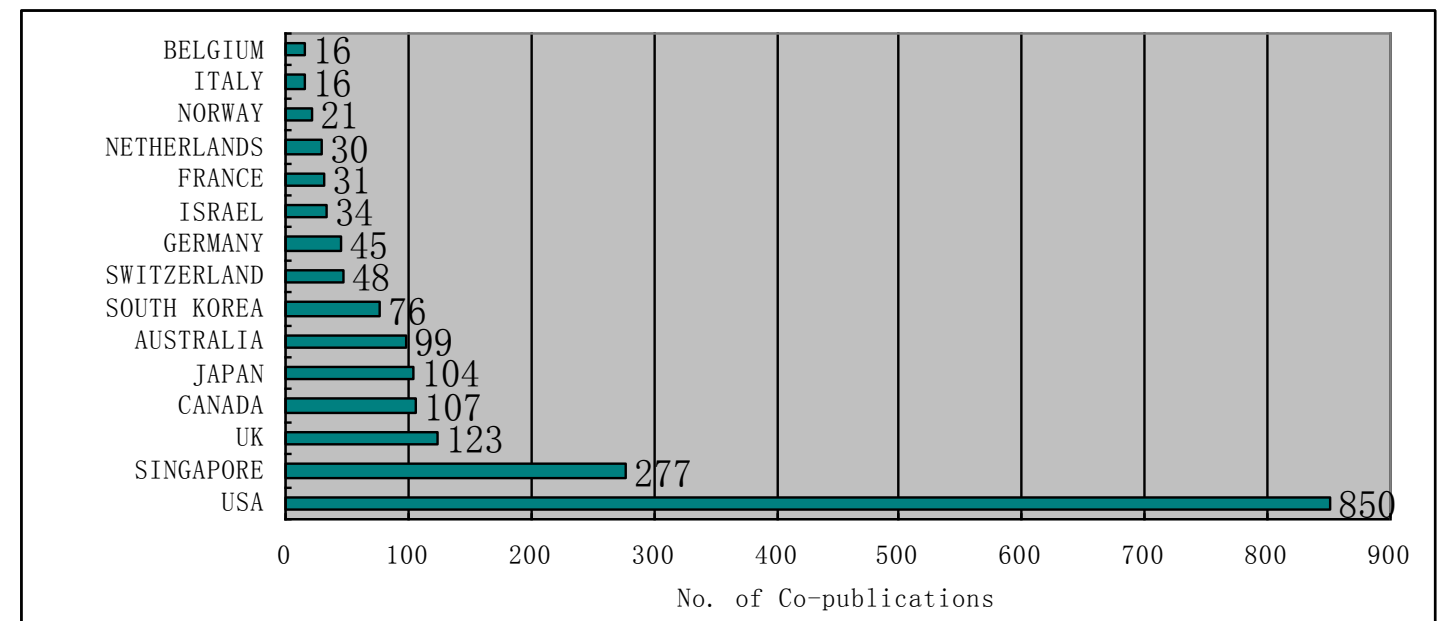

Fig.2 Publications with collaborative country during 2003-2012 
From Figure2 the characteristics of UESTC with collaboration nations and regions can be seen:

$>$ During 2003-2012, the total of co-publication with United States held in the first because United States is the position of today's world center of science and technology.

$>$ UESTC has extensive international collaboration with other countries and regions and the international collaboration countries and regions with UESTC are mainly in western developed countries.

$>$ Partner in Asia, mainly in Singapore, Japan and South Korea, among them the most closely collaboration country is Singapore. The total of co-publication with Singapore ranks the second place and It was known as "Chinese phenomenon"(Jin Bihui, 2007).

\subsection{Scientific Disciplines}

The count of scientific publications in certain fields of research shows the emergence of university strengths in particular areas. From Table3, it can be well observed that the scientific productivity of UESTC emanates from physics with the highest percentage of publications (38.51\%), followed by engineering (34.89\%), Materials Science $(14.17 \%)$, computer Science $(11.47 \%)$ and optics $(11.08 \%)$.

Table3 Publications under Top15 scientific disciplines during 2003-2012

\begin{tabular}{|c|l|c|c|}
\hline Rank & Scientific Disciplines & No.of publication & Percentage(\%) \\
\hline 1 & PHYSICS & 3100 & 38.51 \\
\hline 2 & ENGINEERING & 2809 & 34.89 \\
\hline 3 & MATERIALS SCIENCE & 1141 & 14.17 \\
\hline 4 & COMPUTER SCIENCE & 923 & 11.47 \\
\hline 5 & OPTICS & 892 & 11.08 \\
\hline 6 & TELECOMMUNICATIONS & 744 & 9.24 \\
\hline 7 & MATHEMATICS & 651 & 8.09 \\
\hline 8 & CHEMISTRY & 561 & 6.97 \\
\hline 9 & SCIENCE TECHNOLOGY OTHER & 352 & 4.37 \\
\hline 10 & TOPICS & 219 & 2.72 \\
\hline 11 & AUTOMATION CONTROL SYSTEMS & 209 & 2.60 \\
\hline 12 & OPERATIONS RESEARCH & 201 & 2.50 \\
\hline 13 & INSTRUMENTS INSTRUMENTATION & 152 & 1.89 \\
\hline 14 & NEUROSCIENCES NEUROLOGY & 123 & \\
\hline 15 & MECHANICS & 90 & \\
\hline
\end{tabular}

In bibliometrics, the citation of publication is an important index to evaluate the quality of the publication (Huang Ningyan et al., 2002).If the time of citation is more, then the influence of the publication is greater. From the previous data we can see the total of co-publication of UESTC during the past decade is increasing year by year. But the quality or influence of the publication also need to pay attention.

In this paper use the relative citation impact (RCI) to analyze the quality of publication in different disciplines. RCI is a relative index. It can reflect the quality of publication in a given disciplines at the same time 
eliminate the disadvantages of the different citation due to the various disciplines and citation habits. RCI compares the citation rate of sector output with the relevant given field's average. It is calculated by dividing the average number of citations per sector publication in a given field/subfield by the average number of citations for all publications in that field/subfield. Thus, a relative citation impact of more than 1.0 (RCI > 1) indicates a higher/better position than the given field's average, while a relative citation impact of less than $1.0(\mathrm{RCI}<1)$ would indicate a relatively low performance. different disciplines has different comparative advantage. Ranking in three former disciplines is neurosciences neurology, science technology other topics and instruments instrumentation. The scientific disciplines of engineering, computer science and optics $(\mathrm{RCI}<1)$ showed the low influence, although the quantity of publication with these disciplines is in the five former disciplines.

From Fig.3, the quality of publication of UESTC in

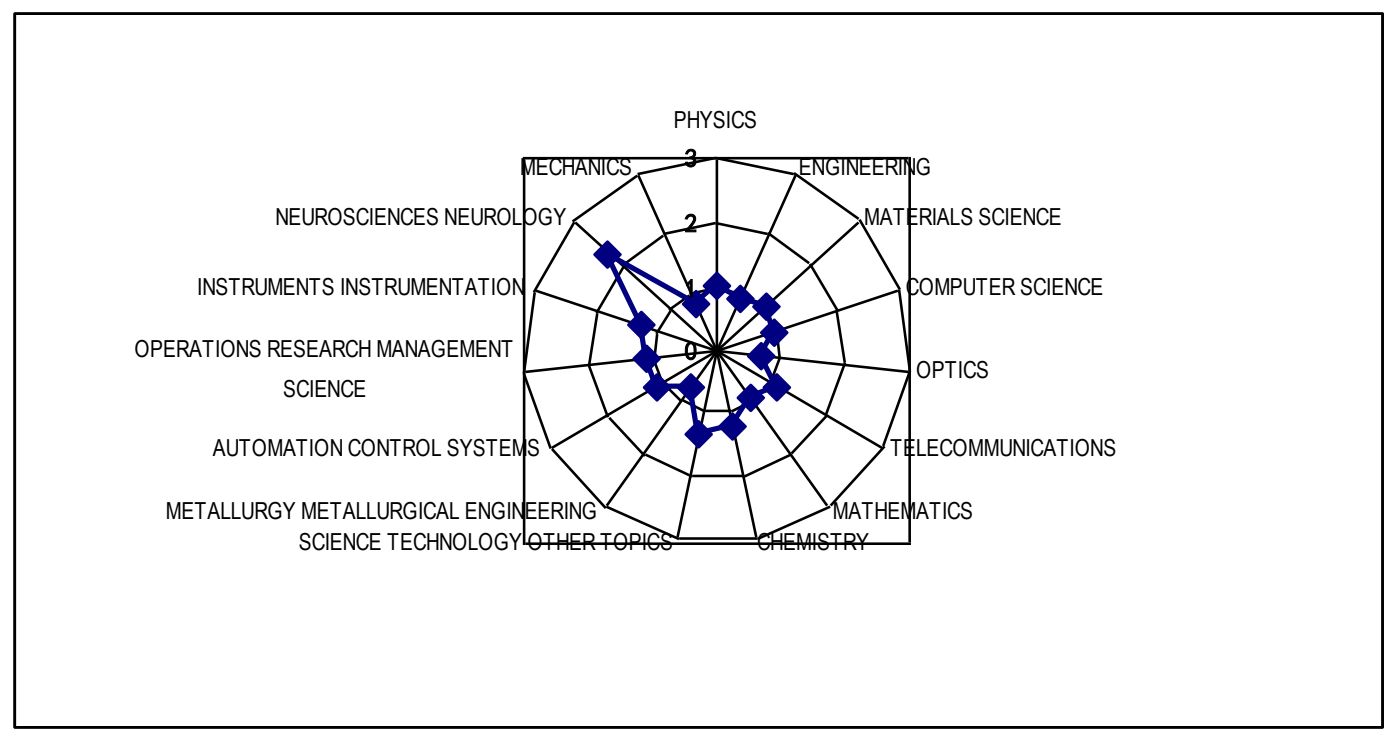

Fig.3 RCI of Top 15 scientific disciplines during 2003-2012

\section{Conclusion}

The followings are the key findings of the present study:

$>$ The total of publication of UESTC during 2003 to 2012 is 8050 and its annual growth rate is average $31.36 \%$.

$>$ Maximum number of four and more than four authored papers (59.89\%) published which is followed by three authors $(24.62 \%)$, two authors (13.18\%), etc.

$>$ The number of international co-publication of UESTC from 2003 to 2012 present growth trend, and the international collaborative countries and regions with UESTC are mainly in western developed countries. In Asia, Singapore, Japan and South Korea have more international collaboration with UESTC, and Singapore plays an important role of collaboration.

$>$ Physics is the highest percentage of publications (38.51\%), followed by engineering (34.89\%), materials science $(14.17 \%)$, computer science (11.47\%) and optics(11.08\%) etc, but neurosciences neurology has the highest relative citation impact.

\section{References}

[1] Almind TC, Ingwersen P. Informetric analyses on the World Wide Web: methodological approaches to webometrics. Journal of Documentation, 53(4), pp404-26, 1997.

[2] Campanario, J.M., González, L. and Rodríguez, C. Structure of the impact factor of academic journals in the field of Education and Educational Psychology: citations 
from editorial board members. Scientometrics, 69(1), pp37-56, 2006.

[3] Falagas, M.E., Karavasiou, A.I. and Bliziotis, I.A. A bibliometric analysis of global trends of research productivity in tropical medicine. Acta Trop, 99, pp 155-159, 2006.

[4] Kumari, L. Trends in synthetic organic chemistry research: cross-country comparison of activity index. Scientometrics, 67, pp467-476, 2006.

[5] Moed, H.F., Burger, W.J.M., Frankfort, J.G. and Vanraan, A.F.J. The use of bibliometric data for the measurement of university research performance. Research Policy, 14(3), pp131-49,1985.

[6] Schubert, A, Glänzel, W. and Braun, T. Scientometric datafiles: a comprehensive set of indicators on 2649 journals and 96 countries in all major science fields and subfields 1981-1985. Scientometrics, 16 (1-6),pp 3-478,1989.

[7] Vergidis, P.I., Karavasiou, A.I., Paraschakis, K., Bliziotis, I.A. and Falagas, M.E. Bibliometric analysis of global trends for research productivity in microbiology. European Journal of Clinical Microbiology; 24, pp342-345, 2005.

[8] Price, D.J. de S. Little Science, Big Science. New York: Columbia University Press, 1963.

[9] Glänzel, W. Co-authorship patterns and trends in the sciences (1980-1998): A bibliometric study with implications for database indexing and search strategies. Library Trends, 50(3), pp461-73, 2002.

[10] Jin Bihui, Zhang Wang, Zhou Qiuju, Yang Liying, Yang Liangbin, Wang Dan, The Internaional S\&T Collaboration of China: The Overses Chinese Phenomenon, Science Focus. 06 ,pp20-27,2007.

[11] Huang Ningyan, Wu Yishan, A comparative study over $\mathrm{S} \& \mathrm{~T}$ output indicators between mainland China and Taiwan region, Studies in Science of Science.06,pp 604-607,2007.

[12] http://www.webofknowledge.com 